\title{
STUDY OF PERCEIVED SOCIAL SUPPORT AND PSYCHOLOGICAL WELL-BEING AMONG PEOPLE LIVING WITH HIV AND AIDS
}

\author{
R. Muralitharan 1 , A. Niranjana Devi², R. Sharmila ${ }^{3}$
}

${ }_{1}^{1}$ Associate Professor, Department of Psychiatry, M. G. Memorial Government Hospital (MGMGH) and KAPV Government Medical College, Tiruchirappalli, Tamilnadu.

2Professor, Department of Psychiatry, M. G. Memorial Government Hospital (MGMGH) and KAPV Government Medical College, Tiruchirappalli, Tamilnadu.

${ }_{3}^{3}$ ART Medical Officer, ART Centre, M. G. Memorial Government Hospital (MGMGH) and KAPV Government Medical College, Tiruchirappalli, Tamilnadu.

ABSTRACT
BACKGROUND
People living with HIV and AIDS (PLWHA) face challenges including lack of social support and mental health issues like
psychological well-being and quality of life. Coping with HIV may be facilitated by their social support network. Perceived social
support might act as a buffer to stress-related crises and may aid in psychological well-being.
The aim of this study is to study perceived social support and psychological well-being among people living with HIV and AIDS.

\section{MATERIALS AND METHODS}

60 PLWHA irrespective of WHO-clinical staging, ART status and duration of illness were subjected for the study. Socio-demographic data, Kuppuswamy's revised socioeconomic status scale, Multidimensional scale of perceived social support and Psychological general well-being index (PGWBI) were administered to study population. Data were analysed using appropriate statistical methods to measure percentage, mean and correlation.

Setting- Study was done in the Department of Psychiatry in association with ART Centre, Mahatma Gandhi Memorial Hospital attached to KAPV Government Medical College, Trichy.

Study Design- This is a cross-sectional - observational study.

\section{RESULTS}

In total, 60 study subjects Males and Females participated in equal number. Study reveals no significant relationship between sociodemographic profile and perceived social support. Psychological well-being had significant relationship with occupation and treatment status of an individual. Study infers there was statistically significant relationship between perceived social support and psychological well-being of an individual.

\section{CONCLUSION}

With participants from different stages and duration of illness, the perceived social support of individuals had been significantly associated with psychological well-being. These findings signify the need for the health care providers to address mental, social support needs and optimise HIV related health outcome.

\section{KEYWORDS}

Psychological Well-Being, Perceived Social Support, PLWHA.

HOW TO CITE THIS ARTICLE: Muralitharan R, Devi AN, Sharmila R. Study of perceived social support and psychological wellbeing among people living with HIV and AIDS. J. Evolution Med. Dent. Sci. 2018;7(01):58-66, DOI: 10.14260/jemds/2018/15

\section{BACKGROUND}

HIV/ AIDS is one of the major and challenging health problems in the contemporary world. India is experiencing rapid spread of HIV infection and falls among the countries, which have highest prevalence of persons living with HIV/ AIDS. People living with HIV and AIDS require care for almost entire lifespan and longevity of the individual is increased significantly following novel ART.

'Financial or Other Competing Interest': None.

Submission 16-11-2017, Peer Review 13-12-2017,

Acceptance 18-12-2017, Published 01-01-2018.

Corresponding Author:

Dr. A. Niranjana Devi,

Professor of Psychiatry,

M. G. Memorial Government Hospital (MGMGH),

and KAPV Government Medical College,

Tiruchirappalli-620017,

Tamilnadu.

E-mail: niranjana.danikrish@gmail.com

DOI: $10.14260 /$ jemds/2018/15

\section{(c) (i) $(9)$}

AIDS is a disease that involves both physical and mental health along with its social consequences because of the stigma, discrimination and attitude of society which can affect their QOL not only from physical health but also from mental and social health and cause problems in interest and activities of the patients. ${ }^{1}$

Due to improved management and treatment, chronic illnesses increase demand for treatment, care and support. In order to fulfil demand for care and management family members, friends and the significant persons in the community can be major sources of support. ${ }^{2}$ Studies suggested the importance of social support as an important factor for influences on well-being and quality of life.3,4 There are ample evidences from previous research that social network and support can improve psychological and physical wellness of the individual by increasing motivation and involvement in treatment, facilitate their living and reduces transmission of disease. 
Social support means care, help a person receives from other people in a close relationship. ${ }^{5}$ Members of family, in particular the spouse, is most important for the association between social support and health. ${ }^{6}$

The social support constitutes emotional, tangible and informational support, each one is distinct since their functions may not be interchangeable.7,8

In countries where no basic infrastructure for their people, individuals and families affected by a health-related issue have no structure to rely on; therefore, well-being is seriously impaired until the ill family member is socially reintegrated. Sociologist Durkheim established the link between diminishing social ties and an increase in suicide. ${ }^{9}$

The perceived social support is described as the extent to which an individual perceives that his/ her needs for support, care, information and feedback are fulfilled by friends and by family and an individual's social competence probably plays a role in the maintenance of his/ her support network.

The social support can either come from family and friends or community-based support, government organisations and the health care facility, ${ }^{10,11}$ peer counselors and health workers are important in providing information for livelihood to receive treatment and counselling in the process of fostering hope. ${ }^{12}$ The support from friends and family is valuable to counter stigma. ${ }^{13}$

The Quality of Life as defined by the WHO is individual's perceptions of their self with respect to culture and values with relation to their goals, standards and expectations. ${ }^{14,15}$ This definition emphasises the overall subjective feeling of happiness, morale and satisfaction. ${ }^{16}$ It imply the degree to which a person's life is desirable or undesirable. ${ }^{17}$

Psychological well-being denotes a selective affective and cognitive aspect of the more general well-being. The observations are directed towards affective or emotional experiences of an intrapersonal nature. 18

The perception of Psychological well-being is not a function of only physical health but is dependent on factors such as age, sex, educational level and income and employment status, independent of the health status. ${ }^{19,20}$

\section{Study Design}

This is a cross-sectional - observational study.

\section{MATERIALS AND METHODS}

The study was carried out on patients diagnosed with HIV and AIDS attending ART Centre, Mahatma Gandhi Memorial Government Hospital (MGMGH) attached to KAPV Government Medical College, Tiruchirapalli. Ethical clearance was obtained from college ethical committee.

\section{Inclusion Criteria}

1. Study subjects who were above 18 years of age.

2. Patients with confirmed HIV test positivity, irrespective of duration of illness and clinical staging of illness.

\section{Exclusion Criteria}

1. Patients with severe cognitive impairment and physical illness.

2. Patients below the age of 18 years.

This study included total of 60 patients, of whom 30 were males and 30 were females and a purposive sampling done. Informed consent was obtained from all participants. This study involved assessment of subjects once.

\section{Measures}

Socio-demographic data sheet was used to record profile of individual participants. Kuppuswamy's socio-economic status scale ${ }^{21}$ was applied.

\section{Multidimensional Scale of Perceived Social Support (MSPSS).}

In present study, Multidimensional Scale of Perceived Social Support ${ }^{22}$ was used to measure social support. It is a 12 -item scale with three subscales of Family (Fam), Friends (Fri) and Significant Others (SO) with equal number of items. The response format is 7 point Likert scale ranging from 1 strongly disagree to 7 strongly agree with maximum score of $84 .{ }^{23}$ The reliability value of $\alpha$ is 0.8953

\section{Psychological General Well-Being Index}

The Psychological General Well-Being Index ${ }^{18}$ (PGWBI) was developed for the evaluation of perceived well-being and distress. It includes six dimensions: Anxiety, Depression, Positive Well-Being, Self-Control, General Health and Vitality. As the subscales are internally consistent, the 22 items have been used with maximal score of 110 . It is a general measure of subjective well-being and hence not condition specific. The reliability value of $\alpha$ is 0.9008 .

Statistical Analyses: Descriptive statistical data were analysed by Percentage, Mean and Standard Deviation. Results were analysed using Chi-square test for qualitative variables, Student T-test, One-Way ANOVA applied to compare various dimensions and Karl Pearson coefficient correlation test was done to compare relationship between perceived social support and psychological well-being.

\section{RESULTS}

In the total study population $(n=60)$, there were equal number of males $(n=30)$ and females $(n=30)$. The minimum age of study population was 22 and maximum age was 65 with the mean age of $42.43 \pm 10.23$.

In this study, persons with different stages of illness with equal participation of males and females were analysed. The mean duration of illness was $4.68 \pm 3.74$, for males mean duration was $4.00 \pm 3.47$ and for females it was $5.36 \pm 3.9$. Regarding socio-economic status, majority (80\%) were from lower/ upper lower class and others from middle/ lower middle (Table 1 and 2).

One-third of study population were widowhood, and in married persons $43 \%(n=16)$ of spouses are infected with HIV. In this study population $80 \%(n=48)$ were on antiretroviral treatment (ART), 86.7\% $(n=26)$ of females are under ART.

Analysing perceived social support, $80 \%(n=48)$ of study population perceived low social support and remaining $20 \%$ experienced medium social support with equal contribution from males and females in both groups. Regarding age group and social support, half of persons between $51-60$ yrs., nearly one-third in 18 - 30 yrs. perceived moderate social support and in all ages above majority experienced low social support. In Joint family setup $16 \%$ and in nuclear family $23 \%$ experienced medium support.

In this study there was no statistical significant association between socio-demographic factors namely age, sex, education, socioeconomic class, marital status, living 
arrangement, area of living and perceived social support level (Table 3).

Assessing Psychological well-being $36.7 \% \quad(n=22)$ expressed positive well-being, $21.7 \%(n=13)$ experienced moderate distress and $41.7 \% \quad(n=25)$ had severe psychological distress. $43.3 \%$ of male and $30 \%$ of females experienced positive well-being and $46.7 \%$ of females and $36.7 \%$ of males expressed severe distress.

Associating age group and well-being more than half of 18 to 30 yrs. and 51 to 60 yrs. groups expressed positive wellbeing. In comparison only one-third of people between 31 50 yrs. of age exhibited positive well-being. This study reveals that people in middle adulthood find it difficult to cope up with the stress of living with HIV.

More than one-third of study subjects in lower/ upper lower class expressed positive well-being and in comparison, more than half of middle/ lower middle class had severe distress. There was no statistical significant relationship between socio-economic class and psychological well-being.

Analysing occupation of study participants with psychological well-being, statistical significant relationship was made out $(\mathrm{p}=0.003)$.
Regarding marital status and well-being 38\% ( $n=14)$ of married persons $(n=37)$ had positive well-being and equal number expressed severe distress, in unmarried persons $75 \%$ $(n=3)$ had severe distress (Table 5).

$41.7 \%$ of the persons on ART felt positive well-being, whereas $16.7 \%$ of persons not on ART experienced positive well-being respectively and this was a statistically significant finding (Table 6). This finding reveals importance of ART on positive well-being and improve the long-term health outcome.

Family living arrangements, area of living, different stages of illness did not have significant relationship with psychological well-being of study populations.

Assessing the association between the perceived social support and psychological well-being of study population in persons with low social support $(n=48)$, nearly half $(n=25)$ had severe psychological distress, $27 \%(n=13)$ was having positive psychological wellbeing in comparison. Majority $(n=9)$ of persons with medium social support $(n=12)$ had positive well-being and one-fourth $(n=3)$ had moderate distress and this finding was significant (Table 6).

\begin{tabular}{|c|c|c|c|}
\hline \multirow{2}{*}{ Sl. No. } & & \multicolumn{2}{|c|}{ Total } \\
\hline & & $(n=60)$ & $100 \%$ \\
\hline \multirow[t]{6}{*}{1} & Age & & \\
\hline & Below 30 & 7 & $11.7 \%$ \\
\hline & 31 to 40 yrs. & 18 & $30.0 \%$ \\
\hline & 41 to 50 yrs. & 25 & $41.7 \%$ \\
\hline & 51 to 60 yrs. & 7 & $11.7 \%$ \\
\hline & 60 to 70 yrs. & 3 & $5 \%$ \\
\hline \multirow{3}{*}{2} & Sex & & \\
\hline & Male & 30 & $50 \%$ \\
\hline & Female & 30 & $50 \%$ \\
\hline & & & \\
\hline \multirow[t]{5}{*}{3} & Education & & \\
\hline & Illiterate & 12 & $20 \%$ \\
\hline & Primary School & 17 & $28.3 \%$ \\
\hline & Middle School & 17 & $28.3 \%$ \\
\hline & Diploma & 14 & $23.3 \%$ \\
\hline \multirow{6}{*}{4} & Occupation & & \\
\hline & Unemployed & 5 & $8.3 \%$ \\
\hline & Unskilled Worker & 9 & $15 \%$ \\
\hline & Semi-Skilled Worker & 29 & $48.3 \%$ \\
\hline & Skilled Worker & 14 & $23.3 \%$ \\
\hline & Clerical and Farmer & 3 & $5 \%$ \\
\hline \multirow[t]{6}{*}{5} & Income & & \\
\hline & Below Rs. 1600 & 8 & $13.3 \%$ \\
\hline & Rs. 1601 to 4809 & 28 & $46.7 \%$ \\
\hline & Rs. 4810 to 8009 & 15 & $25.0 \%$ \\
\hline & Rs. 8010 to 12019 & 8 & $13.3 \%$ \\
\hline & Rs. 16020 to 32049 & 1 & $1.7 \%$ \\
\hline \multirow{3}{*}{6} & Socio-Economic Status & & \\
\hline & Middle/ Lower Middle & 12 & $20 \%$ \\
\hline & Lower/ Upper Lower & 48 & $80 \%$ \\
\hline \multirow{4}{*}{7} & Marital Status & & \\
\hline & Never Married & 4 & $6.7 \%$ \\
\hline & Married & 37 & $61.7 \%$ \\
\hline & Widow/ Widower & 19 & $31.7 \%$ \\
\hline
\end{tabular}




\begin{tabular}{|c|c|c|c|}
\hline 8 & Living Status & \\
\hline & Nuclear Family & 35 & $58.3 \%$ \\
\hline & Joint Family & 25 & $41.7 \%$ \\
\hline & & & \\
\hline & Area of Living & 39 & $35 \%$ \\
\hline & Rural & 21 & \\
\hline & Urban & 53 & $88.3 \%$ \\
\hline & Religion & 4 & $6.7 \% \%$ \\
\hline
\end{tabular}

\begin{tabular}{|c|c|c|c|c|c|c|}
\hline Sl. No. & Item & $\mathbf{N}$ & Min. & Max. & Mean & S.D \\
\hline 1 & Age & 60 & 22 & 65 & 42.43 & 10.235 \\
\hline 2 & CD-4 Count & 60 & 102 & 1215 & 475.92 & 266.163 \\
\hline 3 & Duration of Illness & 60 & .50 & 14.00 & 4.6833 & 3.74388 \\
\hline 4 & MSPSS Sig. Others & 60 & 4 & 19 & 9.62 & 4.203 \\
\hline 5 & MSPSS Family & 60 & 7 & 24 & 16.50 & 4.367 \\
\hline 6 & MSPSS Friends & 60 & 4 & 21 & 10.27 & 5.118 \\
\hline 7 & MSPSS Total Score & 60 & 19 & 60 & 36.38 & 10.750 \\
\hline 8 & PWBI Anxiety & 60 & 7 & 22 & 14.75 & 4.413 \\
\hline 9 & PWBI Depressed Mood & 60 & 3 & 14 & 9.13 & 2.758 \\
\hline 10 & PWBI Positive Well-Being & 60 & 6 & 18 & 11.85 & 3.323 \\
\hline 11 & PWBI Self-Control & 60 & 4 & 14 & 9.30 & 2.533 \\
\hline 12 & PWBI General Health & 60 & 4 & 15 & 9.28 & 2.436 \\
\hline 13 & PWBI Vitality & 60 & 5 & 20 & 11.73 & 3.714 \\
\hline 14 & PWBI Grand Total Score & 60 & 45 & 97 & 66.03 & 13.745 \\
\hline 15 & Age at which Patient comes to know about illness & 60 & 21 & 63 & 37.85 & 10.066 \\
\hline \multicolumn{7}{|c|}{ Table 2. Descriptive Statistics } \\
\hline
\end{tabular}

\begin{tabular}{|c|c|c|c|c|c|c|c|}
\hline \multirow{3}{*}{$\begin{array}{c}\text { Socio-Demographic } \\
\text { Variables }\end{array}$} & \multicolumn{6}{|c|}{ MSPSS } & \multirow{3}{*}{ Statistical Inference } \\
\hline & \multicolumn{2}{|c|}{ Low Support } & \multicolumn{2}{|c|}{ Moderate Support } & \multicolumn{2}{|c|}{ Total } & \\
\hline & $\mathbf{n}$ & $\%$ & $\mathbf{n}$ & $\%$ & $\mathbf{n}$ & $\%$ & \\
\hline \multicolumn{7}{|c|}{ Age } & \multirow{6}{*}{$\begin{array}{c}\mathrm{X}^{2}=9.454 \mathrm{Df}=4.051>0.05 \\
\text { Not Significant }\end{array}$} \\
\hline 18 to 30 yrs. & 5 & $10.4 \%$ & 2 & $16.7 \%$ & 7 & $11.7 \%$ & \\
\hline 31 to 40 yrs. & 17 & $35.4 \%$ & 1 & $8.3 \%$ & 18 & $30.0 \%$ & \\
\hline 41 to 50 yrs. & 20 & $41.7 \%$ & 5 & $41.7 \%$ & 25 & $41.7 \%$ & \\
\hline 51 to 60 yrs. & 3 & $6.3 \%$ & 4 & $33.3 \%$ & 7 & $11.7 \%$ & \\
\hline 61 to 70 yrs. & 3 & $6.3 \%$ & 0 & $.0 \%$ & 3 & $5.0 \%$ & \\
\hline \multicolumn{7}{|c|}{ Sex } & \multirow{3}{*}{$\begin{array}{c}\mathrm{X}^{2}=0.000 \mathrm{Df}=11.000>0.05 \\
\text { Not Significant }\end{array}$} \\
\hline Male & 24 & $50.0 \%$ & 6 & $50.0 \%$ & 30 & $50.0 \%$ & \\
\hline Female & 24 & $50.0 \%$ & 6 & $50.0 \%$ & 30 & $50.0 \%$ & \\
\hline \multicolumn{7}{|c|}{ Socio Economic Class } & \multirow{3}{*}{$\begin{array}{c}\mathrm{X}^{2}=0.234 \mathrm{Df}=1.628>0.05 \\
\text { Not Significant }\end{array}$} \\
\hline Middle/ Lower Middle & 9 & $18.8 \%$ & 3 & $25.0 \%$ & 12 & $20.0 \%$ & \\
\hline Lower/ Upper Lower & 39 & $81.3 \%$ & 9 & $75.0 \%$ & 48 & $80.0 \%$ & \\
\hline \multicolumn{7}{|c|}{ Religion } & \multirow{4}{*}{$\begin{array}{c}\mathrm{X}^{2}=0.831 \mathrm{Df}=2.660>0.05 \\
\text { Not Significant }\end{array}$} \\
\hline Hindu & 42 & $87.5 \%$ & 11 & $91.7 \%$ & 53 & $88.3 \%$ & \\
\hline Muslim & 3 & $6.3 \%$ & 1 & $8.3 \%$ & 4 & $6.7 \%$ & \\
\hline Christian & 3 & $6.3 \%$ & 0 & $.0 \%$ & 3 & $5.0 \%$ & \\
\hline \multicolumn{7}{|c|}{ Marital Status } & \multirow{5}{*}{$\begin{array}{c}\mathrm{X}^{2}=3.060 \mathrm{Df}=3.382>0.05 \\
\text { Not Significant }\end{array}$} \\
\hline Never Married & 4 & $8.3 \%$ & 0 & $.0 \%$ & 4 & $6.7 \%$ & \\
\hline Married & 31 & $64.6 \%$ & 6 & $50.0 \%$ & 37 & $61.7 \%$ & \\
\hline Widow & 10 & $20.8 \%$ & 5 & $41.7 \%$ & 15 & $25.0 \%$ & \\
\hline Widower & 3 & $6.3 \%$ & 1 & $8.3 \%$ & 4 & $6.7 \%$ & \\
\hline \multicolumn{7}{|c|}{ Living Arrangement } & \multirow{3}{*}{$\begin{array}{c}\mathrm{X}^{2}=0.429 \mathrm{Df}=1.513>0.05 \\
\text { Not Significant }\end{array}$} \\
\hline Joint Family & 21 & $43.8 \%$ & 4 & $33.3 \%$ & 25 & $41.7 \%$ & \\
\hline Nuclear Family & 27 & $56.3 \%$ & 8 & $66.7 \%$ & 35 & $58.3 \%$ & \\
\hline \multicolumn{7}{|c|}{ Area of Living } & \multirow{3}{*}{$\begin{array}{c}\mathrm{X}^{2}=3.590 \mathrm{Df}=1.058>0.05 \\
\text { Not Significant }\end{array}$} \\
\hline Rural & 34 & $70.8 \%$ & 5 & $41.7 \%$ & 39 & $65.0 \%$ & \\
\hline Urban & 14 & $29.2 \%$ & 7 & $58.3 \%$ & 21 & $35.0 \%$ & \\
\hline \multicolumn{8}{|c|}{ Table 3. Association between MSPSS and Socio-D } \\
\hline
\end{tabular}




\begin{tabular}{|c|c|c|c|c|c|c|c|}
\hline \multirow{3}{*}{ Variables } & \multicolumn{6}{|c|}{ MSPSS } & \multirow{3}{*}{ Statistical Inference } \\
\hline & \multicolumn{2}{|c|}{ Low Support } & \multicolumn{2}{|c|}{ Moderate Support } & \multicolumn{2}{|r|}{ Total } & \\
\hline & $\mathbf{n}$ & $\%$ & $\mathbf{n}$ & $\%$ & $\mathbf{n}$ & $\%$ & \\
\hline \multicolumn{7}{|c|}{ Stage of Illness } & \multirow{4}{*}{$\begin{array}{c}\mathrm{X}^{2}=0.754 \mathrm{Df}=2.686>0.05 \\
\text { Not Significant }\end{array}$} \\
\hline Stage - I & 15 & $31.3 \%$ & 5 & $41.7 \%$ & 20 & $33.3 \%$ & \\
\hline Stage - II & 15 & $31.3 \%$ & 4 & $33.3 \%$ & 19 & $31.7 \%$ & \\
\hline Stage - III & 18 & $37.5 \%$ & 3 & $25.0 \%$ & 21 & $35.0 \%$ & \\
\hline \multicolumn{7}{|c|}{ Duration of Illness } & \multirow{7}{*}{$\begin{array}{c}\mathrm{X}^{2}=4.375 \mathrm{Df}=5.497>0.05 \\
\text { Not Significant }\end{array}$} \\
\hline 6 months & 8 & $16.7 \%$ & 0 & $.0 \%$ & 8 & $13.3 \%$ & \\
\hline 6 months to 1 year & 8 & $16.7 \%$ & 1 & $8.3 \%$ & 9 & $15.0 \%$ & \\
\hline 1 to 3 yrs. & 7 & $14.6 \%$ & 3 & $25.0 \%$ & 10 & $16.7 \%$ & \\
\hline 3 to 5 yrs. & 8 & $16.7 \%$ & 2 & $16.7 \%$ & 10 & $16.7 \%$ & \\
\hline 5 to 10 yrs. & 14 & $29.2 \%$ & 4 & $33.3 \%$ & 18 & $30.0 \%$ & \\
\hline 10 to 15 yrs. & 3 & $6.3 \%$ & 2 & $16.7 \%$ & 5 & $8.3 \%$ & \\
\hline \multicolumn{7}{|c|}{ PWBI } & \multirow{4}{*}{$\begin{array}{c}\mathrm{X}^{2}=12.338 \mathrm{Df}=2.002<0.05 \\
\text { Significant }\end{array}$} \\
\hline Severe Distress & 25 & $52.1 \%$ & 0 & $.0 \%$ & 25 & $41.7 \%$ & \\
\hline Moderate Distress & 10 & $20.8 \%$ & 3 & $25.0 \%$ & 13 & $21.7 \%$ & \\
\hline Positive Well-Being & 13 & $27.1 \%$ & 9 & $75.0 \%$ & 22 & $36.7 \%$ & \\
\hline \multicolumn{7}{|c|}{ ART } & \multirow{3}{*}{$\begin{array}{c}\mathrm{X}^{2}=1.276 \mathrm{Df}=1.259>0.05 \\
\text { Not Significant }\end{array}$} \\
\hline No & 11 & $22.9 \%$ & 1 & $8.3 \%$ & 12 & $20.0 \%$ & \\
\hline Yes & 37 & $77.1 \%$ & 11 & $91.7 \%$ & 48 & $80.0 \%$ & \\
\hline \multicolumn{7}{|c|}{ Status of Spouse } & \multirow{4}{*}{$\begin{array}{c}\mathrm{X}^{2}=2.614 \mathrm{Df}=2.271>0.05 \\
\text { Not Significant }\end{array}$} \\
\hline Infected with HIV & 15 & $31.3 \%$ & 1 & $8.3 \%$ & 16 & $26.7 \%$ & \\
\hline Not Infected & 16 & $33.3 \%$ & 5 & $41.7 \%$ & 21 & $35.0 \%$ & \\
\hline Not Applicable & 17 & $35.4 \%$ & 6 & $50.0 \%$ & 23 & $38.3 \%$ & \\
\hline Total & 48 & $100.0 \%$ & 12 & $100.0 \%$ & 60 & $100.0 \%$ & \\
\hline \multicolumn{8}{|c|}{ Table 4. Association between MSPSS and Variables } \\
\hline
\end{tabular}

\begin{tabular}{|c|c|c|c|c|c|c|c|c|c|}
\hline \multirow{3}{*}{ Variables } & \multicolumn{8}{|c|}{ PGWBI } & \multirow{3}{*}{$\begin{array}{l}\text { Statistical } \\
\text { Inference }\end{array}$} \\
\hline & \multicolumn{2}{|c|}{ Severe Distress } & \multicolumn{2}{|c|}{ Moderate Distress } & \multicolumn{2}{|c|}{ Positive Well-Being } & \multicolumn{2}{|c|}{ Total } & \\
\hline & $\mathbf{n}$ & $\%$ & $\mathbf{n}$ & $\%$ & $\mathbf{n}$ & $\%$ & $\mathbf{n}$ & $\%$ & \\
\hline \multicolumn{9}{|c|}{ Age } & \multirow{6}{*}{$\begin{array}{c}X^{2}=9.531 \\
D f=8.300>0.05 \\
\text { Not Significant }\end{array}$} \\
\hline 18 to 30 yrs. & 3 & $12.0 \%$ & 0 & $.0 \%$ & 4 & $18.2 \%$ & 7 & $11.7 \%$ & \\
\hline 31 to 40 yrs. & 9 & $36.0 \%$ & 4 & $30.8 \%$ & 5 & $22.7 \%$ & 18 & $30.0 \%$ & \\
\hline 41 to 50 yrs. & 11 & $44.0 \%$ & 6 & $46.2 \%$ & 8 & $36.4 \%$ & 25 & $41.7 \%$ & \\
\hline 51 to 60 yrs. & 0 & $.0 \%$ & 3 & $23.1 \%$ & 4 & $18.2 \%$ & 7 & $11.7 \%$ & \\
\hline 61 to 70 yrs. & 2 & $8.0 \%$ & 0 & $.0 \%$ & 1 & $4.5 \%$ & 3 & $5.0 \%$ & \\
\hline \multicolumn{9}{|c|}{ Sex } & \multirow{3}{*}{$\begin{array}{c}X^{2}=1.164 \\
D f=2.559>0.05 \\
\text { Not Significant }\end{array}$} \\
\hline Male & 11 & $44.0 \%$ & 6 & $46.2 \%$ & 13 & $59.1 \%$ & 30 & $50.0 \%$ & \\
\hline Female & 14 & $56.0 \%$ & 7 & $53.8 \%$ & 9 & $40.9 \%$ & 30 & $50.0 \%$ & \\
\hline \multicolumn{9}{|c|}{ Occupation } & \multirow{6}{*}{$\begin{array}{c}\mathrm{X}^{2}=23.458 \\
\mathrm{Df}=8.003<0.05 \\
\text { Significant }\end{array}$} \\
\hline Unemployed & 2 & $8.0 \%$ & 0 & $.0 \%$ & 3 & $13.6 \%$ & 5 & $8.3 \%$ & \\
\hline Unskilled Worker & 2 & $8.0 \%$ & 6 & $46.2 \%$ & 1 & $4.5 \%$ & 9 & $15.0 \%$ & \\
\hline Semi-Skilled Worker & 15 & $60.0 \%$ & 2 & $15.4 \%$ & 12 & $54.5 \%$ & 29 & $48.3 \%$ & \\
\hline Skilled Worker & 3 & $12.0 \%$ & 5 & $38.5 \%$ & 6 & $27.3 \%$ & 14 & $23.3 \%$ & \\
\hline $\begin{array}{c}\text { Clerical, Shop Owner, } \\
\text { Farmer }\end{array}$ & 3 & $12.0 \%$ & 0 & $.0 \%$ & 0 & $.0 \%$ & 3 & $5.0 \%$ & \\
\hline \multicolumn{9}{|c|}{ Socio-Economic Class } & \multirow{3}{*}{$\begin{array}{c}\mathrm{X}^{2}=2.276 \\
\mathrm{Df}=2.320>0.05 \\
\text { Not Significant }\end{array}$} \\
\hline Middle/ Lower Middle & 7 & $28.0 \%$ & 1 & $7.7 \%$ & 4 & $18.2 \%$ & 12 & $20.0 \%$ & \\
\hline Lower/Upper Lower & 18 & $72.0 \%$ & 12 & $92.3 \%$ & 18 & $81.8 \%$ & 48 & $80.0 \%$ & \\
\hline \multicolumn{9}{|c|}{ Marital Status } & \multirow{5}{*}{$\begin{array}{c}X^{2}=3.702 \\
\text { Df }=6.717>0.05 \\
\text { Not Significant }\end{array}$} \\
\hline Never Married & 3 & $12.0 \%$ & 0 & $.0 \%$ & 1 & $4.5 \%$ & 4 & $6.7 \%$ & \\
\hline Married & 14 & $56.0 \%$ & 9 & $69.2 \%$ & 14 & $63.6 \%$ & 37 & $61.7 \%$ & \\
\hline Widow & 6 & $24.0 \%$ & 4 & $30.8 \%$ & 5 & $22.7 \%$ & 15 & $25.0 \%$ & \\
\hline Widower & 2 & $8.0 \%$ & 0 & $.0 \%$ & 2 & $9.1 \%$ & 4 & $6.7 \%$ & \\
\hline \multicolumn{9}{|c|}{ Living Arrangement } & \multirow{3}{*}{$\begin{array}{c}\quad \mathrm{X}^{2}=0.141 \\
\mathrm{Df}=2.932>0.05 \\
\text { Not Significant }\end{array}$} \\
\hline Joint Family & 10 & $40.0 \%$ & 6 & $46.2 \%$ & 9 & $40.9 \%$ & 25 & $41.7 \%$ & \\
\hline Nuclear Family & 15 & $60.0 \%$ & 7 & $53.8 \%$ & 13 & $59.1 \%$ & 35 & $58.3 \%$ & \\
\hline \multicolumn{9}{|c|}{ Area of Living } & \multirow{4}{*}{$\begin{array}{c}\mathrm{X}^{2}=1.267 \\
\mathrm{Df}=2.531>0.05 \\
\text { Not Significant }\end{array}$} \\
\hline Rural & 18 & $72.0 \%$ & 7 & $53.8 \%$ & 14 & $63.6 \%$ & 39 & $65.0 \%$ & \\
\hline Urban & 7 & $28.0 \%$ & 6 & $46.2 \%$ & 8 & $36.4 \%$ & 21 & $35.0 \%$ & \\
\hline \multicolumn{9}{|c|}{ Table 5. Relationship between PGWBI and Socio-Demographic Variables } & \\
\hline
\end{tabular}




\begin{tabular}{|c|c|c|c|c|c|c|c|c|c|}
\hline \multirow{3}{*}{ Variables } & \multicolumn{8}{|c|}{ PGWBI } & \multirow{3}{*}{ Statistical Inference } \\
\hline & \multicolumn{2}{|c|}{ Severe Distress } & \multicolumn{2}{|c|}{ Moderate Distress } & \multicolumn{2}{|c|}{ Positive Well-Being } & \multicolumn{2}{|c|}{ Total } & \\
\hline & $\mathbf{n}$ & $\%$ & $\mathbf{n}$ & $\%$ & $\mathbf{n}$ & $\%$ & $\mathbf{n}$ & $\%$ & \\
\hline \multicolumn{9}{|c|}{ Stage of Illness } & \multirow{4}{*}{$\begin{array}{c}\mathrm{X}^{2}=3.162 \mathrm{Df}=4.531>0.05 \\
\text { Not Significant }\end{array}$} \\
\hline Stage - I & 6 & $24.0 \%$ & 5 & $38.5 \%$ & 9 & $40.9 \%$ & 20 & $33.3 \%$ & \\
\hline Stage - II & 8 & $32.0 \%$ & 3 & $23.1 \%$ & 8 & $36.4 \%$ & 19 & $31.7 \%$ & \\
\hline Stage - III & 11 & $44.0 \%$ & 5 & $38.5 \%$ & 5 & $22.7 \%$ & 21 & $35.0 \%$ & \\
\hline \multicolumn{9}{|c|}{ CD-4 Count } & \multirow{6}{*}{$\begin{array}{c}\mathrm{X}^{2}=9.923 \mathrm{Df}=8.270>0.05 \\
\text { Not Significant }\end{array}$} \\
\hline Below 250 & 7 & $28.0 \%$ & 1 & $7.7 \%$ & 5 & $22.7 \%$ & 13 & $21.7 \%$ & \\
\hline 251 to 500 & 9 & $36.0 \%$ & 8 & $61.5 \%$ & 8 & $36.4 \%$ & 25 & $41.7 \%$ & \\
\hline 501 to 750 & 5 & $20.0 \%$ & 2 & $15.4 \%$ & 7 & $31.8 \%$ & 14 & $23.3 \%$ & \\
\hline 751 to 1000 & 1 & $4.0 \%$ & 2 & $15.4 \%$ & 2 & $9.1 \%$ & 5 & $8.3 \%$ & \\
\hline Above 1001 & 3 & $12.0 \%$ & 0 & $.0 \%$ & 0 & $.0 \%$ & 3 & $5.0 \%$ & \\
\hline \multicolumn{9}{|c|}{ Duration of Illness } & \multirow{7}{*}{$\begin{array}{c}\mathrm{X}^{2}=15.394 \mathrm{Df}=10.118 \\
>0.05 \\
\text { Not Significant }\end{array}$} \\
\hline 6 months & 5 & $20.0 \%$ & 3 & $23.1 \%$ & 0 & $.0 \%$ & 8 & $13.3 \%$ & \\
\hline 6 months to 1 year & 4 & $16.0 \%$ & 2 & $15.4 \%$ & 3 & $13.6 \%$ & 9 & $15.0 \%$ & \\
\hline 1 to 3 yrs. & 2 & $8.0 \%$ & 2 & $15.4 \%$ & 6 & $27.3 \%$ & 10 & $16.7 \%$ & \\
\hline 3 to 5 yrs. & 5 & $20.0 \%$ & 4 & $30.8 \%$ & 1 & $4.5 \%$ & 10 & $16.7 \%$ & \\
\hline 5 to 10 yrs. & 8 & $32.0 \%$ & 1 & $7.7 \%$ & 9 & $40.9 \%$ & 18 & $30.0 \%$ & \\
\hline 10 to 15 yrs. & 1 & $4.0 \%$ & 1 & $7.7 \%$ & 3 & $13.6 \%$ & 5 & $8.3 \%$ & \\
\hline \multicolumn{9}{|c|}{ ART } & \multirow{3}{*}{$\begin{array}{c}\mathrm{X}^{2}=7.444 \mathrm{Df}=2.024<0.05 \\
\text { Significant }\end{array}$} \\
\hline No & 4 & $16.0 \%$ & 6 & $46.2 \%$ & 2 & $9.1 \%$ & 12 & $20.0 \%$ & \\
\hline Yes & 21 & $84.0 \%$ & 7 & $53.8 \%$ & 20 & $90.9 \%$ & 48 & $80.0 \%$ & \\
\hline \multicolumn{9}{|c|}{ Status of Spouse } & \multirow{4}{*}{$\begin{array}{c}\mathrm{X}^{2}=2.429 \mathrm{Df}=4.657>0.05 \\
\text { Not Significant }\end{array}$} \\
\hline Infected with HIV & 8 & $32.0 \%$ & 3 & $23.1 \%$ & 5 & $22.7 \%$ & 16 & $26.7 \%$ & \\
\hline Not Infected & 6 & $24.0 \%$ & 6 & $46.2 \%$ & 9 & $40.9 \%$ & 21 & $35.0 \%$ & \\
\hline Not Applicable & 11 & $44.0 \%$ & 4 & $30.8 \%$ & 8 & $36.4 \%$ & 23 & $38.3 \%$ & \\
\hline \multicolumn{9}{|c|}{ MSPSS Score } & \multirow{3}{*}{$\begin{array}{c}\mathrm{X}^{2}=12.338 \mathrm{Df}=2.002<0.05 \\
\text { Significant }\end{array}$} \\
\hline Low Acuity & 25 & $100.0 \%$ & 10 & $76.9 \%$ & 13 & $59.1 \%$ & 48 & $80.0 \%$ & \\
\hline Moderate Acuity & 0 & $.0 \%$ & 3 & $23.1 \%$ & 9 & $40.9 \%$ & 12 & $20.0 \%$ & \\
\hline Total & 25 & $100.0 \%$ & 13 & $100.0 \%$ & 22 & $100.0 \%$ & 60 & $100.0 \%$ & \\
\hline \multicolumn{10}{|c|}{ Table 6. Relationship between PGWBI and Variables } \\
\hline
\end{tabular}

\begin{tabular}{|c|c|c|c|}
\hline $\begin{array}{c}\text { MSPSS } \\
\text { Dimensions }\end{array}$ & $\begin{array}{c}\text { Male (n= 30) } \\
\text { Mean } \pm \text { S.D }\end{array}$ & $\begin{array}{c}\text { Female (n= 30) } \\
\text { Mean } \pm \text { S.D }\end{array}$ & Significance \\
\hline 1. Significant Others & $9.83 \pm 4.36$ & $9.40 \pm 4.09$ & $\mathrm{t}=.396 \mathrm{df}=58.693>0.05^{*}$ \\
\hline 2. Family & $16.20 \pm 4.38$ & $16.80 \pm 4.40$ & $\mathrm{t}=-.529 \mathrm{df}=58.599>0.05^{*}$ \\
\hline 3. Friends & $11.00 \pm 4.96$ & $9.53 \pm .5 .25$ & $\mathrm{t}=1.112 \mathrm{df}=58.271>0.05^{*}$ \\
\hline 4. MSPSS Overall Score & $37.03 \pm 10.55$ & $35.73 \pm 11.08$ & $\mathrm{t}=.465 \mathrm{df}=58.644>0.05^{*}$ \\
\hline \multicolumn{2}{|r}{ Table 7. Comparison of Perceived Social Support (MSPSS) among Male and Female } \\
\hline
\end{tabular}

*Not Significant

\begin{tabular}{|l|c|c|c|}
\hline \multicolumn{1}{|c|}{ PWBI Dimensions } & Male (n= 30) Mean \pm S.D & Female (n= 30) Mean \pm S.D & Significance \\
\hline 1. Anxiety & $15.17 \pm 4.80$ & $14.33 \pm 4.02$ & $\mathrm{t}=.728 \mathrm{df}=580.469>0.05^{*}$ \\
\hline 2. Depressed Mood & $9.47 \pm 2.60$ & $8.80 \pm 2.90$ & $\mathrm{t}=.935 \mathrm{df}=580.354>0.05^{*}$ \\
\hline 3. Positive Well-Being & $12.70 \pm 3.42$ & $11.00 \pm 3.04$ & $\mathrm{t}=2.033 \mathrm{df}=580.047<0.05($ Significant) \\
\hline 4. Self-Control & $9.40 \pm 2.66$ & $9.20 \pm 2.44$ & $\mathrm{t}=.303 \mathrm{df}=580.763>0.05^{*}$ \\
\hline 5. General Health & $9.00 \pm 2.58$ & $9.57 \pm 2.28$ & $\mathrm{t}=-.899 \mathrm{df}=580.372>0.05^{*}$ \\
\hline 6. Vitality & $11.83 \pm 3.99$ & $11.63 \pm 3.47$ & $\mathrm{t}=.207 \mathrm{df}=58.837>0.05^{*}$ \\
\hline 7. PWBI- Overall Score & $67.57 \pm 14.28$ & $64.50 \pm 13.25$ & $\mathrm{t}=.862 \mathrm{df}=58.392>0.05^{*}$ \\
\hline \multicolumn{2}{|r|}{ Table 8. Comparison of Psychological Well-Being (PWBI) among Male and Female } \\
\hline
\end{tabular}

*Not Significant

\begin{tabular}{|c|c|c|c|c|c|c|}
\hline \multicolumn{2}{|c|}{} & $\begin{array}{c}\text { Stage-I } \\
(\mathbf{n = 2 0 )}\end{array}$ & $\begin{array}{c}\text { Stage-II } \\
(\mathbf{n = 1 9 )}\end{array}$ & $\begin{array}{c}\text { Stage-III } \\
(\mathbf{n = 2 1})\end{array}$ & Df & Statistical Inference \\
\hline \multirow{3}{*}{ MSPSS } & Significant Others & $10.30 \pm 4.414$ & $9.68 \pm 3.931$ & $8.90 \pm 4.323$ & $2 / 57$ & $\mathrm{f}=.560 .575>0.05^{*}$ \\
\cline { 2 - 7 } & Family & $18.70 \pm 4.014$ & $15.95 \pm 4.503$ & $14.90 \pm 3.859$ & $2 / 57$ & $\mathrm{f}=4.590 .014<0.05$ Significant \\
\cline { 2 - 7 } & Friends & $10.80 \pm 5.521$ & $10.42 \pm 5.015$ & $9.62 \pm 4.995$ & $2 / 57$ & $\mathrm{f}=.278 .758>0.05^{*}$ \\
\cline { 2 - 7 } & Total Score & $39.80 \pm 11.261$ & $36.05 \pm 11.138$ & $33.43 \pm 9.373$ & $2 / 57$ & $\mathrm{f}=1.866 .164>0.05^{*}$ \\
\hline
\end{tabular}




\begin{tabular}{|c|c|c|c|c|c|c|}
\hline & Anxiety & $16.30 \pm 4.462$ & $14.89 \pm 4.081$ & $13.14 \pm 4.293$ & $2 / 57$ & $\mathrm{f}=2.796 .069>0.05^{*}$ \\
\cline { 2 - 7 } & Depressed Mood & $9.70 \pm 2.922$ & $9.16 \pm 2.794$ & $8.57 \pm 2.580$ & $2 / 57$ & $\mathrm{f}=.854 .431>0.05^{*}$ \\
\cline { 2 - 7 } PGWBI & Positive Well-Being & $12.70 \pm 3.466$ & $12.00 \pm 3.180$ & $10.90 \pm 3.223$ & $2 / 57$ & $\mathrm{f}=1.551 .221>0.05^{*}$ \\
\cline { 2 - 7 } & Self-Control & $9.30 \pm 2.618$ & $9.16 \pm 2.672$ & $9.43 \pm 2.441$ & $2 / 57$ & $\mathrm{f}=.055 .946>0.05^{*}$ \\
\cline { 2 - 7 } & General Health & $9.70 \pm 2.736$ & $9.37 \pm 2.338$ & $8.81 \pm 2.250$ & $2 / 57$ & $\mathrm{f}=.694 .504>0.05^{*}$ \\
\cline { 2 - 7 } & Vitality & $12.70 \pm 3.526$ & $11.74 \pm 3.724$ & $10.81 \pm 3.816$ & $2 / 57$ & $\mathrm{f}=1.343 .269>0.05^{*}$ \\
\cline { 2 - 6 } & Grand Total Score & $70.35 \pm 14.666$ & $66.32 \pm 14.016$ & $61.67 \pm 11.723$ & $2 / 57$ & $\mathrm{f}=2.129 .128>0.05^{*}$ \\
\hline \multicolumn{7}{|c|}{ Table 9. Association of MSPSS and PGWBI with Stages of IIIness } \\
\hline
\end{tabular}

*Not Significant

\begin{tabular}{|c|c|c|c|c|c|c|c|c|}
\hline & $\begin{array}{c}<6 \text { months } \\
(n=8)\end{array}$ & \begin{tabular}{|c|}
6 months to 1 \\
yr. $(n=9)$
\end{tabular} & $\begin{array}{c}1 \text { to } 3 \text { yrs. } \\
(n=10)\end{array}$ & $\begin{array}{c}3 \text { to } 5 \text { yrs. } \\
(n=10)\end{array}$ & $\begin{array}{c}5 \text { to } 10 \text { yrs. } \\
(n=18)\end{array}$ & $\begin{array}{c}10 \text { to } 15 \text { yrs. } \\
(n=5)\end{array}$ & Df & $\begin{array}{l}\text { Statistical } \\
\text { Inference }\end{array}$ \\
\hline $\begin{array}{l}\text { MSPSS Sig. } \\
\text { Others }\end{array}$ & $7.50 \pm 3.117$ & $7.11 \pm 3.516$ & $13.50 \pm 3.894$ & $8.90 \pm 2.961$ & $9.83 \pm 4.489$ & $10.40 \pm 4.037$ & $5 / 54$ & $\begin{array}{c}\mathrm{f}=3.448 \\
.009<0.05 \\
\text { Significant }\end{array}$ \\
\hline $\begin{array}{l}\text { MSPSS } \\
\text { Family }\end{array}$ & $14.75 \pm 3.955$ & $17.00 \pm 5.000$ & $16.50 \pm 4.197$ & $15.20 \pm 4.050$ & $16.33 \pm 4.243$ & $21.60 \pm 2.702$ & $5 / 54$ & $\begin{array}{c}\mathrm{f}=1.979 \\
.097>0.05^{*}\end{array}$ \\
\hline \begin{tabular}{|c|} 
MSPSS \\
Friends \\
\end{tabular} & $8.38 \pm 3.503$ & $9.11 \pm 5.349$ & $12.20 \pm 5.073$ & $10.70 \pm 6.255$ & $9.94 \pm 4.476$ & $11.80 \pm 6.535$ & $5 / 54$ & $\begin{array}{c}f=.696 \\
.629>0.05^{*} \\
\end{array}$ \\
\hline \begin{tabular}{|c|} 
MSPSS Total \\
Score
\end{tabular} & $30.63 \pm 8.017$ & $33.22 \pm 11.421$ & $42.20 \pm 7.955$ & $34.80 \pm 9.864$ & $36.11 \pm 11.591$ & $43.80 \pm 12.256$ & $5 / 54$ & $\begin{array}{c}\mathrm{f}=1.845 \\
.120>0.05^{*}\end{array}$ \\
\hline $\begin{array}{l}\text { PGWBI } \\
\text { Anxiety }\end{array}$ & $10.75 \pm 4.234$ & $13.22 \pm 3.734$ & $17.50 \pm 4.223$ & $13.80 \pm 3.293$ & $15.61 \pm 4.104$ & $17.20 \pm 4.764$ & $5 / 54$ & $\begin{array}{c}\mathrm{f}=3.430 \\
.009<0.05 \\
\text { Significant }\end{array}$ \\
\hline $\begin{array}{c}\text { PGWBI } \\
\text { Depressed } \\
\text { Mood } \\
\end{array}$ & $8.63 \pm 2.973$ & $9.33 \pm 2.449$ & $11.00 \pm 1.333$ & $8.00 \pm 1.764$ & $8.83 \pm 3.348$ & $9.20 \pm 3.633$ & $5 / 54$ & $\begin{array}{c}\mathrm{f}=1.407 \\
.236>0.05^{*}\end{array}$ \\
\hline $\begin{array}{c}\text { PGWBI } \\
\text { Positive } \\
\text { Well-Being }\end{array}$ & $10.00 \pm 3.295$ & $11.44 \pm 3.745$ & $12.80 \pm 3.011$ & $11.60 \pm 2.319$ & $11.83 \pm 3.485$ & $14.20 \pm 3.899$ & $5 / 54$ & $\begin{array}{c}\mathrm{f}=1.220 \\
.313>0.05^{*}\end{array}$ \\
\hline \begin{tabular}{|c|}
$\begin{array}{c}\text { PGWBI Self- } \\
\text { Control }\end{array}$ \\
\end{tabular} & $10.00 \pm 2.070$ & $9.33 \pm 3.536$ & $9.40 \pm 1.174$ & $9.60 \pm 2.171$ & $8.89 \pm 3.008$ & $8.80 \pm 2.775$ & $5 / 54$ & $\begin{array}{c}\mathrm{f}=.270 \\
.928>0.05^{*}\end{array}$ \\
\hline $\begin{array}{c}\text { PGWBI } \\
\text { General } \\
\text { Health }\end{array}$ & $8.38 \pm 3.068$ & $9.22 \pm 2.167$ & $8.50 \pm 2.121$ & $9.30 \pm 1.829$ & $9.72 \pm 2.824$ & $10.80 \pm 1.789$ & $5 / 54$ & $\begin{array}{c}\mathrm{f}=.929 \\
.469>0.05^{*}\end{array}$ \\
\hline $\begin{array}{l}\text { PGWBI } \\
\text { Vitality } \\
\end{array}$ & $11.00 \pm 4.629$ & $11.56 \pm 4.640$ & $11.30 \pm 2.497$ & $10.50 \pm 2.224$ & $12.11 \pm 4.114$ & $15.20 \pm 2.168$ & $5 / 54$ & $\begin{array}{c}\mathrm{f}=1.249 \\
.300>0.05^{*}\end{array}$ \\
\hline $\begin{array}{c}\text { PGWBI } \\
\text { Grand Total } \\
\text { Score } \\
\end{array}$ & $58.75 \pm 10.011$ & $64.11 \pm 13.495$ & $70.50 \pm 12.039$ & $62.80 \pm 7.714$ & $67.00 \pm 17.436$ & $75.20 \pm 13.971$ & $5 / 54$ & $\begin{array}{c}\mathrm{f}=1.301 \\
.277>0.05^{*}\end{array}$ \\
\hline
\end{tabular}

*Not Significant

\begin{tabular}{|c|c|c|c|c|c|c|c|c|c|c|}
\hline & & & & $\begin{array}{l}\text { PGWBI- } \\
\text { Anxiety }\end{array}$ & $\begin{array}{l}\text { PGWBI } \\
\text { Depressed } \\
\text { Mood }\end{array}$ & $\mid \begin{array}{c}\text { PGWBI } \\
\text { Positive } \\
\text { Well-Being }\end{array}$ & $\begin{array}{l}\text { PGWBI } \\
\text { Self- } \\
\text { Control }\end{array}$ & $\begin{array}{c}\text { PGWBI } \\
\text { General } \\
\text { Health }\end{array}$ & $\begin{array}{l}\text { PGWBI } \\
\text { Vitality }\end{array}$ & \begin{tabular}{|c|} 
PGWBI \\
Grand \\
Total Score
\end{tabular} \\
\hline & Mean & S.D & & $\begin{array}{c}14.75 \pm \\
4.413 \\
\end{array}$ & $\begin{array}{l}9.13 \pm \\
2.758 \\
\end{array}$ & \begin{tabular}{|c|}
$11.85 \pm$ \\
3.323
\end{tabular} & $\begin{array}{l}9.30 \pm \\
2.533 \\
\end{array}$ & $\begin{array}{l}9.28 \pm \\
2.436 \\
\end{array}$ & $\begin{array}{c}11.73 \pm \\
3.714 \\
\end{array}$ & \begin{tabular}{|c|}
$66.03 \pm$ \\
13.745 \\
\end{tabular} \\
\hline \multirow{2}{*}{$\begin{array}{c}\text { MSPSS } \\
\text { Sig. Others }\end{array}$} & \multirow{2}{*}{9.62} & \multirow{2}{*}{4.203} & $\mathrm{r}$ & $.597(* *)$ & $.560(* *)$ & $.571\left(^{* *}\right)$ & $.341(* *)$ & $.300\left(^{*}\right)$ & $.451\left(^{* *}\right)$ & $.678\left(^{* *}\right)$ \\
\hline & & & sig. & .000 & .000 & .000 & .008 & .020 & .000 & .000 \\
\hline \multirow{2}{*}{$\begin{array}{l}\text { MSPSS } \\
\text { Family }\end{array}$} & \multirow{2}{*}{16.50} & \multirow{2}{*}{4.367} & $r$ & $.319\left(^{*}\right)$ & $.332(* *)$ & $.378\left(^{* *}\right)$ & .198 & $.335\left(^{* *}\right)$ & $.508\left(^{* *}\right)$ & $.492(* *)$ \\
\hline & & & sig. & .013 & .010 & .003 & .130 & .009 & .000 & .000 \\
\hline \multirow{2}{*}{$\begin{array}{l}\text { MSPSS } \\
\text { Friends }\end{array}$} & \multirow{2}{*}{10.27} & \multirow{2}{*}{5.118} & $r$ & $.516\left(^{* *}\right)$ & $.392(* *)$ & $.644\left(^{* *}\right)$ & $.404\left(^{* *}\right)$ & $.427(* *)$ & $.516\left(^{* *}\right)$ & $.687(* *)$ \\
\hline & & & sig. & .000 & .002 & .000 & .001 & .001 & .000 & .000 \\
\hline \multirow{2}{*}{$\begin{array}{c}\text { Overall } \\
\text { MSPSS Score }\end{array}$} & \multirow{2}{*}{36.38} & \multirow{3}{*}{10.750} & $r$ & $.609\left(^{* *}\right)$ & $.541\left(^{* *}\right)$ & $.683(* *)$ & $.406\left(^{* *}\right)$ & $.457\left({ }^{* *}\right)$ & $.628\left(^{* *}\right)$ & $.792(* *)$ \\
\hline & & & sig. & .000 & .000 & .000 & .001 & .000 & .000 & .000 \\
\hline & & & $\mathrm{n}$ & 60 & 60 & 60 & 60 & 60 & 60 & 60 \\
\hline
\end{tabular}

${ }^{* *}$ Correlation is significant at the $0.01,{ }^{*}$ Correlation is significant at the 0.05 level.

\section{DISCUSSION}

The main objective of the present study was to examine the role of perceived social support as the predictor of psychological well-being in PLWHA.
On analysing the perceived social support, the mean score in this study was $36.38 \pm 10.75$ (19 to 60 ) with males having higher mean score comparing to females. This is similar to the findings in the studies done by Sushil Yadav, ${ }^{24}$ Klein K et al,25 Nelson Obiora Okonkwo. ${ }^{26}$ There was no statistically 
significant difference between perceived social support from individual groups like significant others, family, friends with respect to gender. Compared to females, males showed higher mean score in perceived social support from friends. The findings of this study show that the mean score of PSS from family is high than from community network, which is similar to the study of Ilebari OA and Fabsoro E27 (Table 7).

There was statistically significant difference found between perceived social support from family and study populations in different stages of illness $(p=0.014)$. Analysing perceived social support in participants with different duration of illness, statistically significant relation was found in the dimension of significant others $(p=0.009)$ and no significance was found with friends and family sub-group and duration of illness (Table 9 and 10).

The mean score of overall psychological well-being in this study was found to be $66.03 \pm 13.74$ ( 45 - 97), for males it was $67.57 \pm 14.28$ and for females it was $64.50 \pm 13.25$. Assessing various dimensions of PWBI, males expressed higher mean scores in the Anxiety, depressed mood, positive well-being dimensions than females and significance among males and females in positive well-being dimensions $(\mathrm{p}=0.047<0.05)$ was made out (Table 8).

In this study, One-Way ANOVA shows significant difference between persons with different duration of illness and anxiety dimension of PWBI ( $p=0.009<0.05)$. No significance was found between study populations with various stages of illness in relation to psychological wellbeing.

In this study, it was found that all persons with severe psychological distress $(n=25)$ were from low perceived social support group. In persons with medium social support, 75\% experienced positive well-being in PGWBI and in comparison $27 \%$ of persons with low support expressed positive wellbeing in PGWBI.

While correlating dimensions of perceived social support and psychological well-being, the result showed there was highly significant relationship in Karl Pearson's correlation test (The correlation Value $(\mathrm{r})=0.792^{* *}$ ) (Table 11), which is in concordance with the study done by Okawa $\mathrm{S}$ and Yasuoka J et al.28 The study findings indicate the importance of social support in maintaining the Psychological well-being.

\section{Limitations}

The study was limited by its cross-sectional design and sample size. Prospective longitudinal design would be more informative about factors studied.

\section{Implications}

In the present era of increased longevity of people living with HIV and AIDS, this study helps in understanding the association between the social support and psychological well-being of the vulnerable individual. These findings should be given importance in intervention programs for this needed population. The study signifies the importance of the connectivity PLWHA has with family members and other social network.

\section{Future Directions}

PLWHA confronts serious mental health problems which impairs quality of life and significantly interfere with physical health. Efforts should be focused on improving the coping style, improving the person's perception of their health condition and social support, to improve their well-being.

\section{CONCLUSION}

This study showed perceived social support was positively associated with psychological well-being of individual. Health professionals and community based workers should provide necessary support and inform PLWHA about psycho-social support from family and friends. Social support may provide an effective resource in vulnerable population with limited resource setting. This will contribute hope, well-being and longevity of the individual.

\section{REFERENCES}

[1] Aranda-Naranjo B. Quality of life in HIV-positive patient: implications and consequences. J Assoc Nurses AIDS Care 2004;15( Suppl 5):S20-S7.

[2] Greenberger E, Chen C, Tally SR, et al. Family, peer and individual correlates of depressive symptomatology among U.S. and Chinese adolescents. Journal of Consulting and Clinical Psychology 2000;68(2):20919.

[3] Ashton E, Vosvick M, Chesney M, et al. Social support and maladaptive coping as predictors of the change in physical health symptoms among persons living with HIV/AIDS. Aids Patient Care and STDs 2005;19(9): 587-98.

[4] Barroso J. Social support and long-term survivors of AIDS. Western Journal of Nursing Research 1997;19(5):554-73.

[5] Ahmed SM, Lemkau JP. Psychosocial influences on health. In: Rakel RE. edr. Textbook of Family medicine. 7th edn. Philadelphia, PA: Saunders Elsevier 2007:35-8.

[6] Bray JH, Campbell TL. The family's influence on health. In: Rakel RE. edr. Textbook of Family medicine. 7th edn. Philadelphia, PA: Saunders Elsevier 2007:25-6.

[7] Leserman J, Perkins DO, Evans DL. Coping with the threat of AIDS: the role of social support. Am J Psychiatry 1992;149(11):1514-20.

[8] Caplan G. Support systems and community mental health: lectures on concept development. Pasadena, CA: Behavioral Publications 1974.

[9] Vaux A, Harrison D. Support network characteristics associated with support satisfaction and perceived support. American Journal of Community Psychology 1985;13(3):245-65.

[10] Shippy RA. Taking care of each other. GMHC Treat Issues 2007;21(2):7-8.

[11] Cutcliffe JR. How do nurses inspire and instil hope interminally ill HIV patients. Journal of Advanced Nursing 1995;22(5):888-95.

[12] Akinsola HA. Fostering hope in people living with AIDS in Africa: the role of primary health-care workers. Australian Journal of Rural Health 2001;9(4):158-65.

[13] Andrews S. Social support as a stress buffer amonghuman immunodeficiency virus-seropositive urban mothers. Holistic Nursing Practice 1995;10(1):36-43.

[14] Whoqol-Bref. Introduction, administration, scoring and generic version of the assessment. Field Trial Version. 1996. WHO, Geneva. Cited on May 12, 2011. http://www.who.int/mental_health/media/en/76.pdf 
[15] Kohli RM, Sane S, Kumar K, et al. Modification of Medical Outcome Study (MOS) instrument for quality of life assessment \& its validation in HIV infected individuals in India. Indian J Med Res 2005;122:297304.

[16] Cummins RA. Comprehensive quality of life scaleadult. Manual. $5^{\text {th }}$ edn. Australia: School of Psychology Deakin University 1997.

[17] Diener E. Guidelines for National Indicators of Subjective Well-Being and Ill-Being. University of Illinois 2005.

[18] Chassany O, Dimenäs E, et al. The Psychological General Well-Being Index (PGWBI) user manual. IQODpsychological general well-being index instruction manual. 2004:43-4.

[19] Louwagie GM, Bachmann MO, Meyer K, et al. Highly active antiretroviral treatment and health related quality of life in South African adults with human immunodeficiency virus infection: a cross-sectional analytical study. BMC Public Health 2007;7:244.

[20] Adedimeji AA, Odutolu O. Care support and quality of life outcomes among persons living with HIV in the HAART era: findings from Southwest Nigeria. World Journal of AIDS 2014;4:1.

[21] Kumar BPR, Dudala SR, Rao AR. Kuppusamy's socioeconomic status scale - a revision of economic parameter for 2012. International Journal of Research \& Development of Health 2013;1(1):2-4.
[22] Zimet GD, Dahlem NW, Zimet SG, et al. The multidimensional scale of perceived social support. Journal of Personality Assessment 1988;52(1):30-41.

[23] Zimet GD, Powell SS, Parley GK, et al. Psychometric characteristics of the multidimensional scale of perceived social support. Journal of Personality Assessment 1990;55(3-4):610-7.

[24] Yadav S. Perceived social support, hope and quality of life of persons living with HIV/AIDS: a case study from Nepal. Qual Life Res 2010;19(2):157-66.

[25] Klein K, Armistead L, Devine D, et al. Socioemotional support in African American families coping with maternal HIV: an examination of mothers' and children's psychosocial adjustment. Behavior Therapy 2000;31(1):1-26.

[26] Okonkwo NO, Larkan F, Galligan M. An assessment of the levels of perceived social support among older adults living with HIV and AIDS in Dublin. Springer Plus 2016;5(1):726.

[27] Ilebari OA, Fabsoro E. Effects of community-based care for people living with HIV/AIDS on their well-being in Benue state. Journal of Social Aspects of HIV/AIDS 2011;4:668-77.

[28] Okawa S, Yasuoka J, Ishikawa N, et al. Perceived social support and psychological well-being of AIDS orphan in urban Kenya. AIDS Care 2011;23(9):1177-85. 